\title{
ФИЗИКАИ АСТРОНОМИЯ
}

DOI: https://doi.org/10.15688/mpcm.jvolsu.2020.4.7

УДК 519.62

Дата поступления статьи: 02.07.2020

ББК 22.193

Дата принятия статьи: 03.11.2020

\section{ОПТИМАЛЬНАЯ ФОРМА РОТОРА ГИРОДИНА В КЛАССЕ КОНИЧЕСКИХ ТЕЛ}

\author{
Андрей Эдуардович Бирюк \\ Кандидат физико-математических наук, начальник отдела \\ дистанционных и вычислительных технологий, \\ Кубанский государственный университет \\ biryuk@kubsu.ru \\ ул. Ставропольская, 149, 350040 г. Краснодар, Российская Федерация

\section{Михаил Иванович Дроботенко} \\ Кандидат физико-математических наук, старший научный сотрудник, \\ Кубанский государственный университет \\ mdrobotenko@mail.ru \\ ул. Ставропольская, 149, 350040 г. Краснодар, Российская Федерация

\section{Игорь Викторович Рядчиков} \\ Кандидат физико-математических наук, заведующий лабораторией \\ робототехники и мехатроники, \\ Кубанский государственный университет \\ ryadchikov@kubsu.ru \\ ул. Ставропольская, 149, 350040 г. Краснодар, Российская Федерация
}

\section{Александр Анатольевич Свидлов}

Кандидат физико-математических наук, научный сотрудник отдела аридных зон (г. Краснодар),

Южный научный центр РАН

svidlov@mail.ru

просп. Чехова, 41, 344006 г. Ростов-на-Дону, Российская Федерация

ISSN 2587-6325. Математ. физика и компьютер. моделирование. 2020. Т. 23. № 4 
Аннотация. Ставится задача поиска оптимальной формы ротора гиродина и угловой скорости вращения, максимизирующие момент импульса относительно оси вращения, с учетом конечной прочности материала. Задача численно решается в классе конических тел вращения. Исследуется зависимость полученного решения от коэффициента Пуассона материала ротора.

Ключевые слова: коэффициент Пуассона, прочность материала, быстро вращающийся диск, краевая задача, экстремальная задача, конечноразностный метод, метод дихотомии.

\section{Введение}

При разработке шагающих роботизированных систем, таких как AnyWalker [2;4], и систем стабилизации их движения на основе гиродинов [3;5] возникает проблема выбора конструкции гиродинов. В частности, необходимо выбрать форму ротора гиродина таким образом, чтобы обеспечить максимальную эффективность работы гиродина. Эффективность обусловливается в основном моментом импульса ротора относительно оси вращения ротора. При фиксированной массе ротора момент импульса можно увеличить за счет увеличения скорости его вращения либо за счет увеличения момента инерции ротора гиродина относительно оси его вращения путем перераспределения массы «подальше» от оси. Как увеличение скорости, так и увеличение момента инерции ведет к увеличению внутренних напряжений в материале ротора. Таким образом, возникает задача выбора скорости вращения и формы ротора гиродина для того, чтобы максимизировать момент импульса при выполнении условий прочности. Поставленную задачу следует рассматривать при дополнительных ограничениях на размер и массу ротора, поскольку эти ограничения являются следствием ограничений на размеры и массу конструкции.

\section{1. Постановка задачи}

Перед тем, как непосредственно перейти к математической постановке задачи исследования, рассмотрим математическую модель упруго-деформированного состояния ротора, приведенную в работе [1].

Ротор гиродина является телом вращения, толщина которого зависит только от расстояния $r$ до оси вращения, $r \in[0, R]$. Поверхность ротора задана вращением кривых $z= \pm z(r)$ вокруг оси. Иными словами, функция $z(r)$ определяет профиль ротора. Толщина ротора на расстоянии $r$ от оси равна $2 z(r)$.

Ротор будем считать тонким, и поэтому пренебрегаем перемещениями и механическими напряжениями в направлении, параллельном оси вращения ротора. В теле ротора образуются поля нормальных напряжений: радиальное $\sigma_{r}$ и кольцевое $\sigma_{t}$. В силу симметрии все силы и напряжения будут функциями расстояния $r$ от оси вращения.

Когда ротор раскручен, он претерпевает деформации, в результате которых произвольная точка $A$ смещается в радиальном направлении в точку $A^{\prime}$ (см. рис. 1$)$.

Поле деформации в точке $A$ определяется вектором $\overrightarrow{A A^{\prime}}$. В силу радиальной симметрии длина этого вектора зависит лишь от радиуса $r=|O A|$. Определим функцию деформации $u:[0, R] \rightarrow \mathbb{R}$, как длину вектора смещения: $u(r)=\left|A A^{\prime}\right|$. Поле деформаций однозначно описывается этой функцией. 


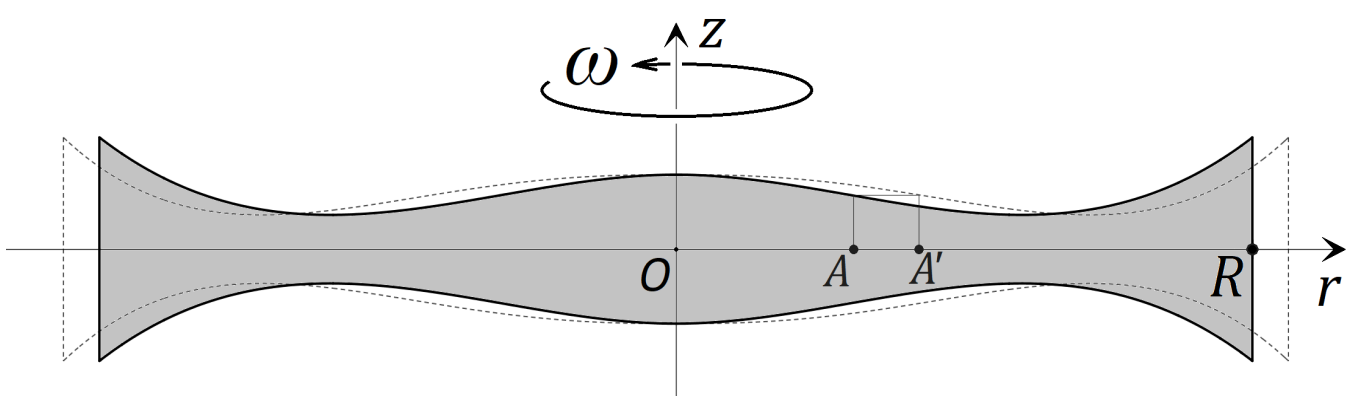

Рис. 1. Профиль ротора и его деформация

Функция $u$ удовлетворяет уравнению упруго-деформируемого состояния [1]:

$$
\frac{E}{1-\mu^{2}}\left(z \frac{d u}{d r}+r \frac{d z}{d r} \frac{d u}{d r}+r z \frac{d^{2} u}{d r^{2}}+\mu \frac{d z}{d r} u-z \frac{u}{r}\right)+\rho \omega^{2} r^{2} z=0, \quad r \in(0 ; R) .
$$

Связь между полем деформаций и полями напряжений описывается уравнениями [1]

$$
\left\{\begin{array}{l}
\sigma_{r}=\frac{E}{1-\mu^{2}}\left(\frac{d u}{d r}+\mu \frac{u}{r}\right), \\
\sigma_{t}=\frac{E}{1-\mu^{2}}\left(\mu \frac{d u}{d r}+\frac{u}{r}\right) .
\end{array}\right.
$$

Здесь $R$ обозначает радиус ротора; $\omega$ - угловая скорость вращения ротора; $\rho$ - плотность; $E-$ модуль Юнга; $\mu-$ коэффициент Пуассона материала ротора.

Положительное значение напряжения $\sigma_{r}$ соответствует растяжению элементарного объема в радиальном направлении, а отрицательное - сжатию. Аналогично знак $\sigma_{t}$ определяет растяжение/сжатие элементарного объема в тангенциальном направлении.

В силу отсутствия воздействия со стороны внешней границы $\sigma_{r}(R)=0$. Ввиду радиальной симметрии центр ротора не смещается, то есть $u(0)=0$. Поэтому уравнение (1) относительно функции $u$ дополняется следующими краевыми условиями:

$$
u(0)=0, \quad \frac{d u}{d r}(R)+\mu \frac{u(R)}{R}=0 .
$$

Краевая задача (1), (3) ставится для каждой положительной на $(0, R)$ функции $z \in$ $\in C^{1}[0, R]$. Классическим решением краевой задачи (1), (3) будем называть функцию

$$
u \in C^{0}[0, R] \cap C^{1}(0, R] \cap C^{2}(0, R),
$$

которая удовлетворяет соотношениям (1) и (3).

Максимальное механическое напряжение, соответствующее растяжению элементарных объемов, в теле ротора, заданного полутолщиной $z$ раскрученного до угловой скорости $\omega$, описывается функционалом:

$$
\mathfrak{S}_{R, \rho, E, \mu}(\omega, z(\cdot))=\sup _{x \in[0, R]} \max \left(\sigma_{r}(x), \sigma_{t}(x)\right) .
$$

В формуле (4) отсутствует модуль, поскольку мы считаем, что разрушение материала может происходить только из-за растяжения. Это обусловливается как постановкой задачи, так и тем, что предел прочности на сжатие существенно выше, чем предел прочности на растяжение.

ISSN 2587-6325. Математ. физика и компьютер. моделирование. 2020. Т. 23. № 4 
При заданном материале, массе $m$ и радиусе $R$ ротора гиродина требуется подобрать его форму $z(r)$ в заданном классе функций $\mathcal{F} \subset C^{1}[0, R]$ с дополнительным условием

$$
4 \pi \rho \int_{0}^{R} r z(r) d r=m
$$

и угловую скорость вращения $\omega$ с целью максимизации момента импульса

$$
L(\omega, z(\cdot))=4 \pi \rho \omega \int_{0}^{R} r^{3} z(r) d r
$$

учитывая при этом конечную прочность материала ротора:

$$
\mathfrak{S}_{R, \rho, E, \mu}(\omega, z(\cdot)) \leqslant[\sigma],
$$

где $[\sigma]$ обозначает максимально допустимое напряжение материала ротора.

Определим множество

$$
K_{R, m, \rho, E,[\sigma], \mu}=\{(\omega, z) \in \mathbb{R} \times \mathcal{F}: \text { выполнены (5) и (6) }\} .
$$

Это множество будем называть множеством, удовлетворяющем условию прочности, или множеством прочности.

Основная задача состоит в том, чтобы найти максимум функционала момента импульса $L(\omega, z(\cdot))$ на множестве $K_{R, m, \rho, E,[\sigma], \mu}$ :

$$
\mathfrak{M}(R, m, \rho, E,[\sigma], \mu)=\max _{(\omega, z(\cdot)) \in K_{R, m, \rho, E,[\sigma], \mu}} L(\omega, z(\cdot)),
$$

предъявить максимизирующую пару $(\omega, z(\cdot)) \in K_{R, m, \rho, E,[\sigma], \mu}$, задающую профиль $z$ оптимального ротора и его оптимальную угловую скорость вращения

В дальнейшем, в настоящей статье, будем считать, что $\rho=7800 \frac{\mathrm{Kr}}{\mathrm{M}^{3}},[\sigma]=147 \mathrm{MПа,}$ $E=200$ ГПа, что соответствует стали. Зафиксируем $m=2 \mathrm{kг,} R=0,12 \mathrm{м}$, что соответствует параметрам разрабатываемой установки [5]. Для упрощения обозначений зависимость от этих пяти параметров в дальнейшем будем опускать. Для стали коэффициент Пуассона $\mu=0,3$, однако, нас интересует вопрос зависимости задачи от параметра $\mu$. Поэтому будем считать, что $\mu \in(-1,1)$, несмотря на то, что изотропных материалов с коэффициентами Пуассона $\mu>0,5$, по-видимому, не существует.

В качестве класса функций $\mathcal{F}$ возьмем многочлены степени не выше первой, задающие конические тела вращения. Для этого класса функций уравнение (1), вообще говоря, не разрешимо аналитически, так же как и в общем случае, что вынуждает нас пользоваться численными методами, даже в этом относительно простом случае. В этом смысле мы не ограничиваем общности предлагаемого подхода. С другой стороны, мы избегаем ряд технических сложностей, например, проверка условия положительности $z(r)$ при $r \in(0, R)$ упрощается, результаты вычислений можно представить более наглядно. Кроме этого, конические роторы проще в изготовлении.

\section{2. Численное решение}

Рассмотрим задачу (7) в классе роторов конической формы. В этом случае полутолщина имеет вид $z(r)=a+b r, 0 \leqslant r \leqslant R$, где $a$ и $b-$ константы. Даже в этом случае 
аналитически решить задачу не удается, кроме некоторых частных случаев, например, $b=0$ или $a=0$. По-видимому, общего аналитического решения в этом классе нет, поэтому будем решать задачу численно.

Из условия (5) следует, что

$$
a=\frac{m}{2 \pi \rho R^{2}}-\frac{2 R}{3} b .
$$

Это, в частности, означает, что при фиксированных $m, \rho$ и $R$ параметры $a$ и $b$ являются зависимыми, то есть класс роторов конической формы может быть описан лишь одним параметром. Выберем для описания параметр $b$, а параметр $a$ будем находить из соотношения связи (8).

Так как $z(0) \geq 0$ и $z(R) \geq 0$, то должны выполняться два условия: $a \geq 0$ и $a+b R \geq 0$, из которых с учетом (8) следует, что

$$
b_{\min }=-\frac{3 m}{2 \pi \rho R^{3}} \leq b \leq b_{\max }=\frac{3 m}{4 \pi \rho R^{3}} .
$$

Поскольку полутолщина $z$ однозначно задается одним параметром $b$, функционал момента импульса $L$ зависит от двух вещественных параметров $\omega$ и $b$ :

$$
L(\omega, b)=4 \pi \rho \omega \int_{0}^{R} r^{3}(a+b r) d r=\frac{1}{2} m \omega R^{2}+\frac{2}{15} b \pi \rho \omega R^{5} .
$$

Задача (7) на классе конических роторов упрощается и принимает вид:

$$
\mathfrak{M}(\mu)=\max _{(\omega, b) \in K_{\mu}} L(\omega, b),
$$

где множество $K_{\mu}$ задает область параметров, удовлетворяющих условию прочности:

$$
K_{\mu}=\left\{(\omega, b) \in[0,+\infty) \times\left[b_{\min }, b_{\max }\right]: \mathfrak{S}_{\mu}(\omega, b) \leqslant[\sigma]\right\} .
$$

Здесь $\mathfrak{S}_{\mu}(\omega, b)$ - функция максимальных напряжений, определяемая выражением (4), в котором $z(r)=a+b r$, параметр $a$ находится из (8).

Значения $(\omega, b)$, обеспечивающие максимум $(11)$, будем называть оптимальными для заданного $\mu$. Ротор соответствующего профиля будем называть оптимальным в классе конических тел.

Мы будем пользоваться фактом, что функции $L(\omega, b)$ и $\mathfrak{S}_{\mu}(\omega, b)$ возрастают при фиксированном $\omega>0$ с возрастанием $b$. Для первой это следует из (10). Для второй функции это утверждение оставляем в качестве гипотезы, основанной на физических рассуждениях, что увеличение $b$ соответствует перераспределению массы ближе к краю с одновременным уменьшением толщины в центре, что должно привести к увеличению максимального напряжения материала ротора. Гипотеза подтверждается численными экспериментами.

Приведем некоторые значения функции $L(\omega, b)$ :

$$
L\left(\omega, b_{\min }\right)=\frac{3}{10} m \omega R^{2}, \quad L(\omega, 0)=\frac{1}{2} m \omega R^{2}, \quad L\left(\omega, b_{\max }\right)=\frac{3}{5} m \omega R^{2} .
$$


Пусть

$$
\Omega_{\mu}=\left\{\omega \geq 0:\{\omega\} \times\left[b_{\min }, b_{\max }\right] \cap K_{\mu} \neq \emptyset\right\}, \quad B_{\mu}(\omega)=\left\{b \in\left[b_{\min }, b_{\max }\right]:(\omega, b) \in K_{\mu}\right\} .
$$

Тогда

$$
\max _{(\omega, b) \in K_{\mu}} L(\omega, b)=\max _{\omega \in \Omega_{\mu}} \max _{b \in B_{\mu}(\omega)} L(\omega, b) .
$$

В силу установленной монотонности функции $L(\omega, b)$ по $b$, внутренний максимум в правой части достигается на значении $b(\omega)=\max B_{\mu}(\omega)$. Используя монотонность функции $\mathfrak{S}_{\mu}(\omega, b)$ по $b$, мы можем численно находить $b(\omega)$ методом дихотомии. Из уравнений (1) и (2) следует факт монотонного возрастания функции $\mathfrak{S}_{\mu}(\omega, b)$ по $\omega$. Поэтому функция $b(\omega)$ монотонно убывает. Это позволяет оптимизировать вычисления при построении области прочности.

Численное значение $\mathfrak{M}(\mu)$ можно найти, например, следующим способом. Для значений параметра $\omega$, начиная от нуля с шагом $\delta \omega>0$ и пока $B_{\mu}(\omega)$ не пусто, находим значения $L(\omega, b(\omega))$, среди которых выбираем наибольшее.

Для проверки условия прочности применялся конечно-разностный подход. Краевая задача (1), (3) дискретизировалась в рамках конечно-разностного подхода следующим образом:

$$
\left\{\begin{array}{l}
u_{0}=0, \\
a_{i} u_{i-1}+b_{i} u_{i}+c_{i} u_{i+1}=f_{i}, \quad i=\overline{1, n-1}, \\
\frac{u_{n}-u_{n-1}}{h}+\mu \frac{u_{n}}{R}=0 .
\end{array}\right.
$$

Здесь $n-$ заданная размерность приближенной задачи; $h=R / n-$ шаг сетки; $r_{i}=i h$, $u_{i}$ - аппроксимация для $u\left(r_{i}\right), i=\overline{0, n} ; a_{i}=A_{i} / h^{2}-B_{i} /(2 h) ; b_{i}=-2 A_{i} / h^{2}+C_{i}$; $c_{i}=A_{i} / h^{2}+B_{i} /(2 h) ; f_{i}=-\rho \omega^{2} r_{i}^{2} z\left(r_{i}\right)\left(1-\mu^{2}\right) / E ; A_{i}=r_{i} z\left(r_{i}\right) ; B_{i}=z\left(r_{i}\right)+r_{i} z^{\prime}\left(r_{i}\right)$; $C_{i}=\mu z^{\prime}\left(r_{i}\right)-z\left(r_{i}\right) / r_{i}$.

При этом конечно-разностные аппроксимации напряжений $\sigma_{r}\left(r_{i}\right)$ и $\sigma_{t}\left(r_{i}\right)$ вычислялись по формулам

где

$$
\begin{gathered}
\sigma_{r, i}=\frac{E}{1-\mu^{2}}\left(\delta u_{i}+\mu \frac{u_{i}}{r_{i}}\right), \quad \sigma_{t, i}=\frac{E}{1-\mu^{2}}\left(\mu \delta u_{i}+\frac{u_{i}}{r_{i}}\right), \quad i=\overline{1, n-1}, \\
\sigma_{r, 0}=\sigma_{t, 0}=\frac{E}{1-\mu} \delta u_{0}
\end{gathered}
$$

$$
\delta u_{0}=\frac{u_{1}-u_{0}}{h}, \quad \delta u_{i}=\frac{u_{i+1}-u_{i-1}}{2 h}, \quad i=\overline{1, n-1}, \quad \delta u_{n}=\frac{u_{n}-u_{n-1}}{h} .
$$

Функцию максимальных напряжений $\mathfrak{S}_{\mu}(\boldsymbol{\omega}, b)$, определяющую область прочности (12), аппроксимируем следующим образом:

$$
\mathfrak{S}_{\mu}^{n}(\boldsymbol{\omega}, b)=\max \left\{\sigma_{r, 0}, \ldots, \sigma_{r, n}, \sigma_{t, 0}, \ldots, \sigma_{t, n}\right\}
$$

\section{3. Частные интегрируемые случаи конических роторов}

В семействе конических роторов можно выделить два интегрируемых случая. Запишем решения для этих случаев и будем их использовать для тестирования сходимости численных решений. При $b=0$ краевая задача (1), (3) имеет решение

$$
u(r)=\frac{\rho \omega^{2} R^{2}(3+\mu)(1-\mu)}{8 E} r-\frac{\rho \omega^{2}\left(1-\mu^{2}\right)}{8 E} r^{3} .
$$


При $a=0$, что соответствует случаю $b=b_{\max }$, краевая задача (1), (3) имеет решение

$$
u(r)=\frac{\rho \omega^{2}\left(1-\mu^{2}\right)(3+\mu) R^{3-s}}{(s+\mu)(11+\mu) E} r^{s}-\frac{\rho \omega^{2}\left(1-\mu^{2}\right)}{(11+\mu) E} r^{3},
$$

где $s=\frac{\sqrt{5-4 \mu}-1}{2}$. Из этого можем получить значения функции максимальных напряжений:

$$
\mathfrak{S}_{\mu}(\omega, 0)=\frac{\max \{3+\mu ; 2-2 \mu\}}{8} \rho \omega^{2} R^{2}, \quad \mathfrak{S}_{\mu}\left(\omega, b_{\max }\right)=\infty .
$$

Замечание 1. Из последнего соотношения следует, что при любом $\mu \in(-1,1)$ для области прочности (12) выполнено включение:

$$
K_{\mu} \subset\{0\} \times\left[b_{\min }, b_{\max }\right] \cup(0,+\infty) \times\left[b_{\min }, b_{\max }\right) .
$$

Для диска равной толщины $(b=0)$ максимальное $\omega$, удовлетворяющее условию прочности (12), задается выражением:

$$
\omega_{D}(\mu)=\frac{1}{R} \sqrt{\frac{8[\sigma]}{\rho \max \{3+\mu ; 2-2 \mu\}}} .
$$

Отсюда находим, что максимальный момент импульса $\mathfrak{M}_{D}(\mu)$ для диска равной толщины, при выполнении условия прочности, имеет следующий вид:

$$
\mathfrak{M}_{D}(\mu)=L\left(\omega_{D}(\mu), 0\right)=\frac{m \omega_{D}(\mu) R^{2}}{2}=m R \sqrt{\frac{2[\sigma]}{\rho \max \{3+\mu ; 2-2 \mu\}}} .
$$

\section{4. Результаты расчетов}

\section{1. Результаты тестирования сходимости}

Приведем результаты тестирования сходимости численного решения к точному в описанных выше интегрируемых случаях.

Оценка погрешности численного метода при $b=0, \mu=0,3$

\begin{tabular}{|c|c|c|c|c|c|c|}
\hline$n$ & 10 & 100 & $10^{3}$ & $10^{4}$ & $10^{5}$ & $10^{6}$ \\
\hline$\frac{\max \left|u\left(r_{i}\right)-u_{i}^{n}\right|}{\max \left|u\left(r_{i}\right)\right|}$ & $1,46 \cdot 10^{-1}$ & $1,48 \cdot 10^{-2}$ & $1,48 \cdot 10^{-3}$ & $1,48 \cdot 10^{-4}$ & $1,48 \cdot 10^{-5}$ & $1,46 \cdot 10^{-6}$ \\
\hline$\frac{\max \left|u^{\prime}\left(r_{i}\right)-\delta u_{i}^{n}\right|}{\max \left|u^{\prime}\left(r_{i}\right)\right|}$ & $9,76 \cdot 10^{-2}$ & $9,19 \cdot 10^{-3}$ & $9,11 \cdot 10^{-4}$ & $9,09 \cdot 10^{-5}$ & $9,09 \cdot 10^{-6}$ & $9,27 \cdot 10^{-7}$ \\
\hline
\end{tabular}

Оценка погрешности численного метода при $b=b_{\max }, \mu=0,25$

\begin{tabular}{|c|c|c|c|c|c|c|}
\hline$n$ & 10 & 100 & $10^{3}$ & $10^{4}$ & $10^{5}$ & $10^{6}$ \\
\hline$\frac{\max \left|u\left(r_{i}\right)-u_{i}^{n}\right|}{\max \left|u\left(r_{i}\right)\right|}$ & $1,35 \cdot 10^{-1}$ & $1,39 \cdot 10^{-2}$ & $1,74 \cdot 10^{-3}$ & $5,63 \cdot 10^{-4}$ & $1,78 \cdot 10^{-4}$ & $5,65 \cdot 10^{-5}$ \\
\hline
\end{tabular}


При других значениях $\mu \in(-1 ; 1)$ результаты аналогичны. Прослеживается сходимость порядка $O(1 / n)$ для $b=0$ и порядка $O\left(1 / n^{s}\right)$ для $b=b_{\max }$. Для $b=b_{\max }$ сходимость более медленная, так как имеет место особенность первой производной точного решения в нуле.

\section{2. Область прочности}

Пользуясь приведенным выше алгоритмом численного решения, рассчитаем область прочности $(12)$ в зависимости от $\mu \in(-1,1)$. Результаты расчетов приведем на рисунке 2. Серым цветом выделена область параметров $\omega$ и $b$, удовлетворяющих условию прочности при $\mu=0,3$. Показаны границы этой области при некоторых других значениях $\mu$.

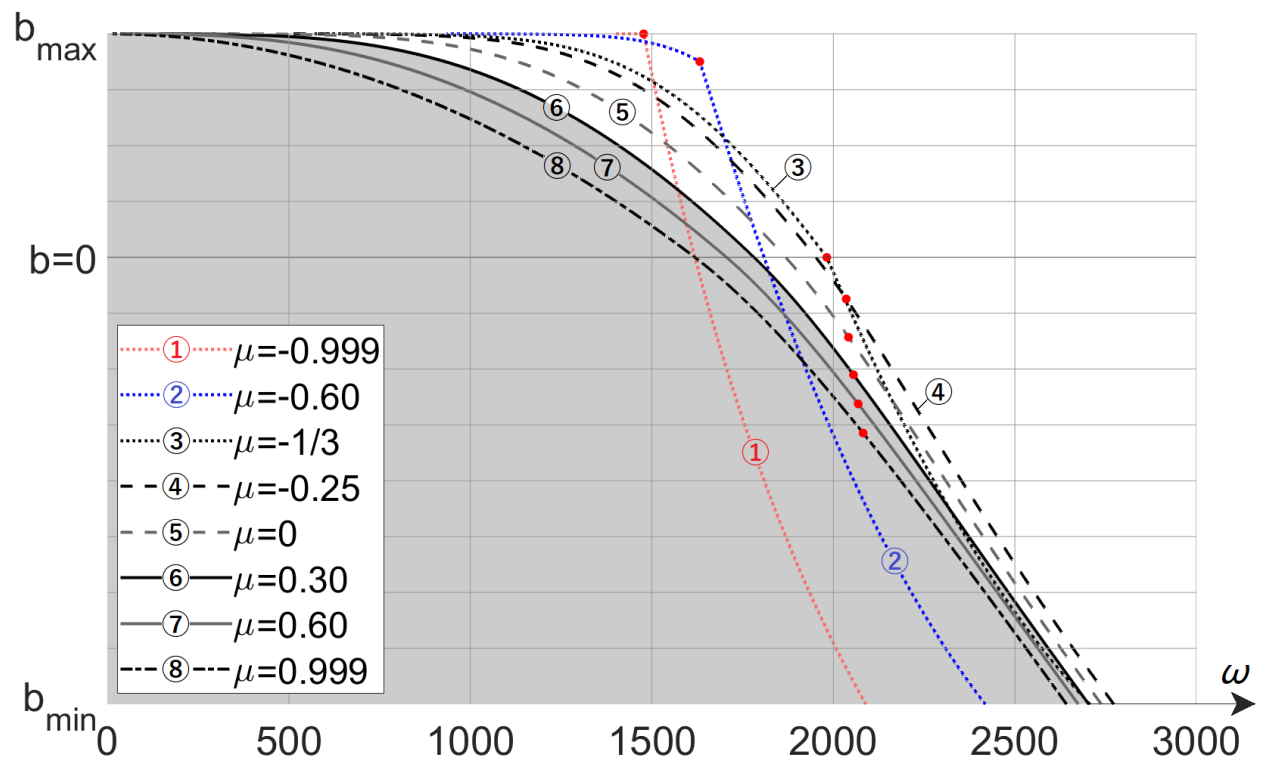

Рис. 2. Область прочности $K_{\mu}$ для некоторых значений $\mu$

На рисунке 2 для каждого из указанных значений $\mu$ выделены точки максимума $(\omega(\mu), b(\mu))$ функционала момента импульса (10) по области $K_{\mu}$. Они перечислены ниже в таблице 3 вместе со значениями целевого функционала $\mathfrak{M}(\mu)=L(\omega(\mu), b(\mu))$ и относительным выигрышем по сравнению с моментом импульса ротора постоянной толщины (14) при заданном $\mu$.

Отметим, что с ростом $\mu$ от -1 до приблизительно $-1 / 4$ область прочности изменяется не монотонно. В частности, она «сокращается» при больших значениях $b$ (около $\left.b_{\max }\right)$, но «растет» при меньших значениях $b$. Около значения $\mu \approx-1 / 4$ характер «немонотонности» усложняется и требует отдельного исследования. Начиная со значения $\mu \approx-1 / 4$ область прочности монотонно убывает с ростом $\mu$.

Значения величин $\omega$ и $\mathfrak{M}$ даны в единицах системы СИ: $\frac{\text { рад }}{c}$ и $\frac{\text { кг.м² }}{c}$ соответственно. Остальные величины являются безразмерными. Справочно приведем значения

$$
b_{\max }=0,0354, \quad b_{\min }=-0,0708 .
$$


Точки максимума момента импульса (10) по $K_{\mu}$ в зависимости от $\mu$

\begin{tabular}{|c|c|c|c|c|c|c|c|c|}
\hline$\mu$ & $-0,999$ & $-0,6$ & $-1 / 3$ & $-0,25$ & 0 & 0,3 & 0,6 & 0,999 \\
\hline$\omega$ & 1477 & 1632 & 1981 & 2036 & 2042 & 2055 & 2068 & 2082 \\
\hline$b$ & 0,0354 & 0,0311 & 0,0000 & $-0,0066$ & $-0,0127$ & $-0,0186$ & $-0,0232$ & $-0,0279$ \\
\hline $\mathfrak{M}$ & 25,5266 & 27,6169 & 28,5335 & 28,2151 & 27,2980 & 26,4854 & 25,8755 & 25,2637 \\
\hline$\frac{\mathfrak{M}-\mathfrak{M}_{D}}{\mathfrak{M}_{D}}$ & $9,5 \%$ & $6,0 \%$ & $0,0 \%$ & $0,4 \%$ & $1,5 \%$ & $3,2 \%$ & $5,4 \%$ & $8,4 \%$ \\
\hline
\end{tabular}

\section{3. Эффективность конических роторов}

Эффективностью оптимального конического ротора при заданном $\mu \in(-1,1)$ по сравнению с ротором постоянной толщины назовем отношение $\frac{\mathfrak{M}(\mu)}{\mathfrak{M}_{D}(\mu)}$ максимального момента импульса (11) к моменту импульса ротора постоянной толщины (14).

На следующем рисунке представлена зависимость эффективности оптимального конического ротора от $\mu \in(-1,1)$.

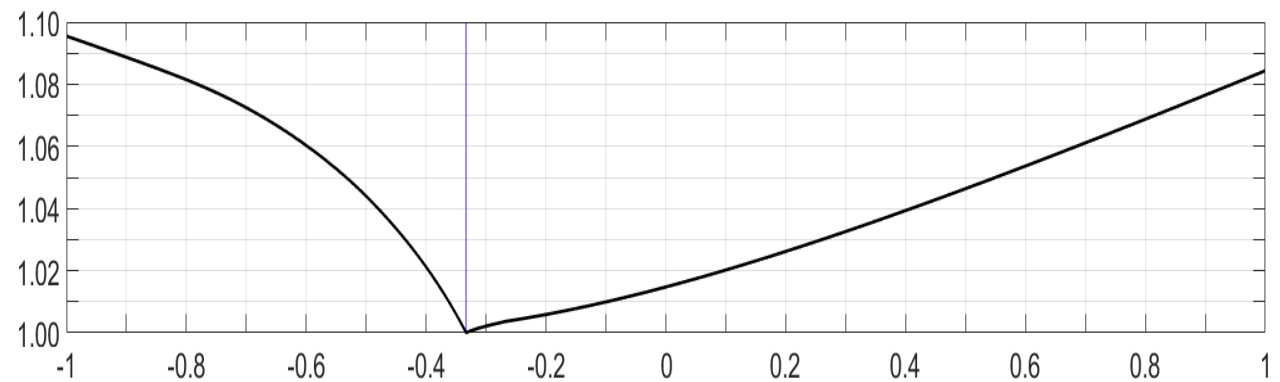

Рис. 3. Выигрыш эффективности оптимального конического ротора по сравнению с диском постоянной толщины

Из графика, приведенного на рисунке 3 , видно, что при значениях $\mu$, близких к \pm 1 , прирост эффективности составляет порядка $9 \%$ относительно диска постоянной ширины, а при значении $\mu=0,3$ прирост эффективности составляет приблизительно $3,2 \%$.

На рисунке 4 показаны графики оптимальной угловой скорости $\omega$ оптимального конического ротора и максимальной угловой скорости $\omega_{D}$ диска постоянной толщины (13), выраженные в $\left[\frac{\mathrm{paz}}{\mathrm{c}}\right]$ в зависимости от $\mu \in(-1,1)$.

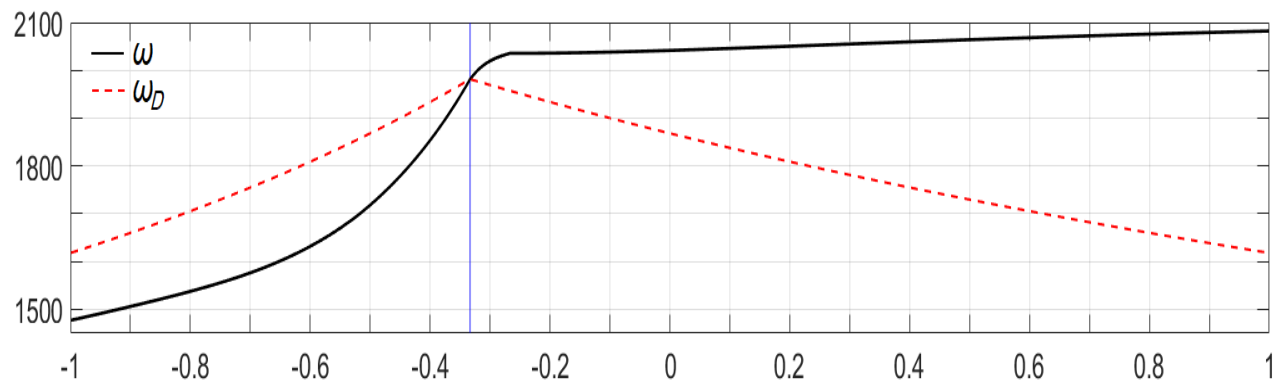

Рис. 4. Значение оптимальной угловой скорости $\omega$ и максимальной угловой скорости $\omega_{D}$ диска постоянной толщины в зависимости от $\mu$

ISSN 2587-6325. Математ. физика и компьютер. моделирование. 2020. Т. 23. № 4 
На рисунке 5 изображен график оптимального наклона профиля $b$, конического ротора (выраженного в безразмерных величинах) в зависимости от коэффициента Пуассона $\mu \in(-1,1)$.

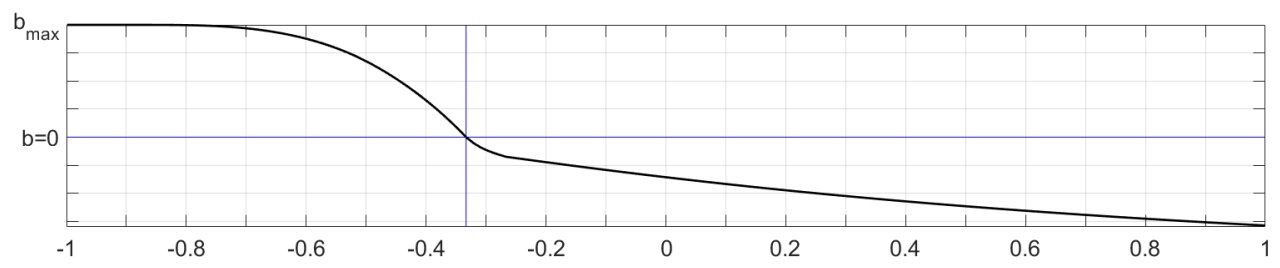

Рис. 5. Значение оптимального наклона $b$ в зависимости от $\mu$

Из рисунка 5 , в частности, следует, что при $\mu>-1 / 3$ толщина оптимального ротора убывает от центра к краю $(b<0)$, а при $\mu<-1 / 3-$ наоборот $(b>0)$.

На рисунках 3-5 отмечено значение $\mu=-1 / 3$, для которого оптимальный ротор совпадает с ротором постоянной толщины.

\section{4. Оптимальная форма стального конического ротора}

Приведем иллюстрацию профиля оптимального конического ротора для $\mu=0,3$ (сталь).

На рисунке 6 серым цветом представлен профиль оптимального конического ротора для $\mu=0,3$. Для наглядности показаны границы профиля диска постоянной толщины (сплошная линия) и границы роторов для $b=b_{\min }$ и $b=b_{\max }$ (прерывистые линии), имеющие ту же массу. Оси градуированы в сантиметрах. В целях читаемости данных масштаб по осям не сохранен.

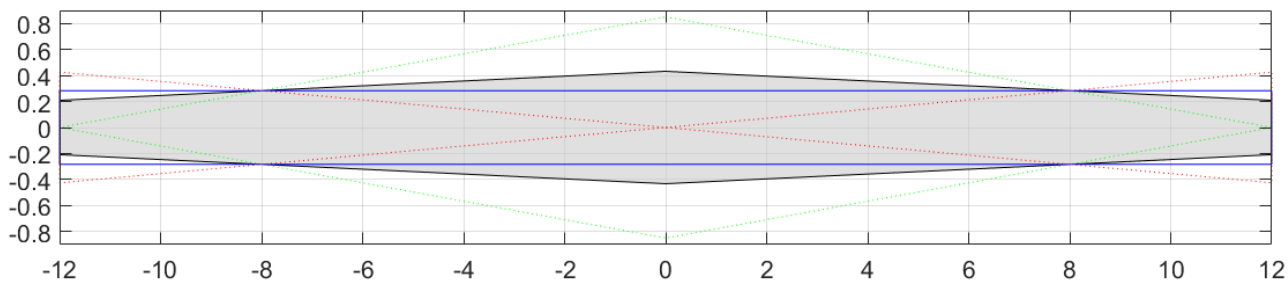

Рис. 6. Форма оптимального конического ротора при $\mu=0,3$

В таблице 4 приведены геометрические характеристики этого оптимального ротора в сравнении с диском равной толщины.

таблица 4

Сравнение оптимального конического ротора с диском для $\mu=0,3$

\begin{tabular}{|c|c|c|}
\hline Характеристика & $\begin{array}{c}\text { Оптимальный конический ротор } \\
\text { для } \mu=0,3\end{array}$ & Диск равной толщины \\
\hline Полутолщина в центре $z(0)$, мм & 4,32 & 2,83 \\
\hline Полутолщина на крае $z(R), \mathrm{mm}$ & 2,09 & 2,83 \\
\hline$z(0) / z(R)$ & 2,068 & 1 \\
\hline Угловая скорость вращения, рад/с & 2055 & 1781 \\
\hline
\end{tabular}




\section{Заключение}

В статье предложен универсальный алгоритм, работающий для широкого набора параметров конструкции и характеристик материалов $(R, m, \rho, E,[\sigma], \mu)$, для нахождения оптимальной формы ротора гиродина в классе конических тел. В случае, рассматриваемом в работе, а именно, для стали при радиусе ротора $R=12 \mathrm{~cm}$, преимущество в моменте импульса найденного конического ротора над ротором постоянной толщины составляет $3 \%$.

Подход к поиску оптимальной формы ротора, предложенный в работе, допускает обобщение / расширение на случай тел вращения более сложной формы, чем конические, но это сопряжено с увеличением вычислительных затрат.

Следует отметить несколько факторов, не учтенных в работе:

- Не рассмотрен способ крепления ротора гиродина к оси вращения и, соответственно, не оценено влияние этого способа крепления на характеристики ротора.

- При прецессии оси вращения ротора изменяется напряженно-деформированное состояние тела ротора и оно будет существенно отличаться от напряженно-деформированного состояния, которое исследовано в работе в рамках приближения «тонкого диска» с осесимметричным полем напряжений и деформаций.

Однако, если учитывать эти факторы, модель становится слишком сложной для эффективного поиска оптимальной формы.

Поставим ряд открытых вопросов и задач.

1) Доказать монотонность функции $\mathfrak{S}(\omega, b)$ по параметру $b$.

2) Получить явную аналитическую оценку для $\omega_{\max }$ такую, что

$$
K_{\mu} \subset\left[0, \omega_{\max }\right] \times\left[b_{\min }, b_{\max }\right] .
$$

3) Исследовать более детально зависимость области прочности $K_{\mu}$ от параметра $\mu$.

4) Исследовать монотонность зависимости оптимальной угловой скорости $\omega$ от значения коэффициента Пуассона $\mu$.

5) Исследовать предельные случаи краевой задачи (1), (3) при $\mu \rightarrow \pm 1$.

6) Рассмотреть случай профиля ротора произвольной формы.

\section{СПИСОК ЛИТЕРАТУРЫ}

1. Миронов, В. М. Конструирование и расчет элементов оборудования отрасли. Ч. II. Толстостенные сосуды и вращающиеся детали / В. М. Миронов, В. М. Беляев. - Томск : Изд-во Том. политех. ун-та, 2003. - 112 с.

2. Разработка конструкции шагающего робота AnyWalker / И. В. Рядчиков, С. И. Сеченев, А. А. Свидлов, А. Э. Бирюк, А. С. Прутский, А. А. Гусев, Е. В. Никульчев // Сборник трудов XIII Всероссийского совещания по проблемам управления ВСПУ-2019. - М. : Изд-во института проблем управления им. В.А. Трапезникова PAH, 2019. - C. 1215-1219. - DOI: https://doi.org/10.25728/vspu.2019.1215.

3. Синтез линейно-квадратичного регулятора для стабилизации обратного маятника гиродином при переносе положения равновесия / И. В. Рядчиков, С. И. Сеченев, Н. В. Михальков, А. Э. Бирюк, А. А. Свидлов, А. А. Гусев, Д. В. Соколов, Е. В. Никульчев // Вестник Рязанского государственного радиотехнического университета. - 2019. № 68. - C. 83-89. - DOI: https://doi.org/10.21667/1995-4565-2019-68-2-83-89. 
4. Constructive solution of the robotic chassis AnyWalker / I. Riadchykov, S. Sechenev, S. Sinitsa, E. Nikulchev // ITM Web of Conferences. - 2016. - Vol. 6. - Article ID: 01003. DOI: https://doi.org/10.1051/itmconf/20160601003.

5. Feedback Control with Equilibrium Revision for CMG-Actuated Inverted Pendulum / I. Riadchykov, S. Sechenev, N. Mikhalkov, A. Biryuk, A. Svidlov, A. Gusev, D. Sokolov, E. Nikulchev // Smart Innovation, Systems and Technologies. - 2020. - Vol. 154. P. 431-440. - DOI: https://doi.org/10.1007/978-981-13-9267-2_35.

\section{REFERENCES}

1. Mironov V.M., Belyaev V.M. Konstruirovanie i raschet elementov oborudovaniya otrasli. Ch. II. Tolstostennye sosudy i vrashchayushchiesya detali [Design and Calculation of Industrial Equipment Elements. Part II. Thick-Walled Vessels and Rotating Parts]. Tomsk, TPU Publ, 2003. 112 p.

2. Ryadchikov I.V., Sechenev S.I., Svidlov A.A., Biryuk A.E., Prutskiy A.S., Gusev A.A., Nikulchev E.V. Razrabotka konstruktsii shagayushchego robota AnyWalker [AnyWalker Walking Robot Design]. Sbornik trudov XIII Vserossiyskogo soveshchaniya po problemam upravleniya VSPU-2019. Moscow, Izd-vo instituta problem upravleniya im. V.A. Trapeznikova RAN, 2019, pp. 1215-1219. DOI: https://doi.org/10.25728/vspu.2019.1215.

3. Ryadchikov I.V., Sechenev S.I., Mikhalkov N.V., Biryuk A.E., Svidlov A.A., Gusev A.A., Sokolov D.V., Nikulchev E.V. Sintez lineyno-kvadratichnogo regulyatora dlya stabilizatsii obratnogo mayatnika girodinom pri perenose polozheniya ravnovesiya [Synthesis of a Linear-Quadratic Regulator for Stabilization of a Pendulum by a Gyrodine While Transferring an Equilibrium Position]. Vestnik Ryazanskogo gosudarstvennogo radiotekhnicheskogo universiteta, 2019, no. 68, pp. 83-89. DOI: https://doi.org/10.21667/1995-4565-2019-68-2-8389.

4. Riadchykov I., Sechenev S., Sinitsa S., Nikulchev E. Constructive Solution of the Robotic Chassis AnyWalker. ITM Web of Conferences, 2016, vol. 6, article ID: 01003. DOI: https://doi.org/10.1051/itmconf/20160601003.

5. Riadchykov I., Sechenev S., Mikhalkov N., Biryuk A., Svidlov A., Gusev A., Sokolov D., Nikulchev E. Feedback Control with Equilibrium Revision for CMG-Actuated Inverted Pendulum. Smart Innovation, Systems and Technologies, 2020, vol. 154, pp. 431-440. DOI: https://doi.org/10.1007/978-981-13-9267-2_35.

\section{OPTIMAL GYRODINE ROTOR SHAPE IN THE CLASS OF CONICAL BODIES}

\section{Andrey E. Biryuk}

Candidate of Physical and Mathematical Sciences, Head of the Department

of Remote and Computing Technologies,

Kuban State University

biryuk@kubsu.ru

Stavropolskaya St, 149, 350040 Krasnodar, Russian Federation

\section{Mikhail I. Drobotenko}

Candidate of Physical and Mathematical Sciences, Senior Researcher,

Kuban State University

mdrobotenko@mail.ru

Stavropolskaya St, 149, 350040 Krasnodar, Russian Federation 


\section{Igor V. Ryadchikov}

Candidate of Physical and Mathematical Sciences, Head of the Laboratory

of Robotics and Mechatronics,

Kuban State University

ryadchikov@kubsu.ru

Stavropolskaya St, 149, 350040 Krasnodar, Russian Federation

\section{Alexander A. Svidlov}

Candidate of Physical and Mathematical Sciences, Researcher,

Department of Arid Zones (Krasnodar),

The Southern Scientific Centre of the Russian Academy of Sciences

svidlov@mail.ru

Chekhova St, 41, 344006 Rostov-on-Don, Russian Federation

Abstract. The problem is to find the optimal shape of the gyrodine rotor and its angular rotation speed that maximizes the angular momentum relative to the axis of rotation at a fixed radius, mass and material of the rotor, taking into account the final strength of the material. The gyrodine rotor is a body of revolution, the thickness of which depends only on the distance $r$ to the axis of rotation, $r \in[0, R]$, where $R$ is the radius of the rotor. The rotor surface is defined by rotating the curves $z= \pm z(r)$ around the axis. When the rotor is spinning, it undergoes deformation due to centrifugal forces. Normal stress fields appear: radial and annular. Assuming the rotor to be thin, deformations can be described by the functions of the radial displacement of the rotor points $u(r)$. Stress fields can be expressed in terms of this function. The functions $u(r)$ and $z(r)$ are related by the equation of the elastically deformed state. This equation is supplied with the boundary conditions for the absence of radial stresses at $r=R$ and the condition for the absence of displacement on the axis of rotation $u(0)=0$. Using the numerical solution of the equation, the problem is solved for the class of conical rotors $z(r)=a+b r$ with two parameters $a$ and $b$. The numerical method is used due to the fact that even in this relatively simple case the problem cannot be solved analytically. Several integrable cases are used to analyze the calculation error in the numerical solution of the problem. The dependence of the problem on the Poisson ratio $\mu \in(-1.1)$ is investigated, with the remaining parameters fixed. The gain in the angular momentum relative to a rotor of constant thickness is compared. The optimal steel conical rotor $(\mu=0.3)$ is 2.068 times thicker at the center than at the edge. Its advantage in the angular momentum over the rotor of constant thickness is $3.2 \%$.

Key words: Poisson's ratio, material strength, rapidly rotating disk, boundary value problem, extremal problem, finite-difference method, dichotomy method. 